\title{
Linx
}

Revue des linguistes de l'université Paris X Nanterre

$10 \mid 1998$

L'indicible et ses marques dans l'énonciation

\section{Dicible et indicible : positions assumées par le sujet du discours}

Utterable and unutterable: roles played by the subject of discourse.

Beth Brait

\section{OpenEdition}

Journals

Édition électronique

URL : http://journals.openedition.org/linx/949

DOI : $10.4000 /$ linx. 949

ISSN : 2118-9692

Éditeur

Presses universitaires de Paris Nanterre

Édition imprimée

Date de publication : 1 juillet 1998

Pagination : 13-20

ISSN : 0246-8743

\section{Référence électronique}

Beth Brait, « Dicible et indicible : positions assumées par le sujet du discours », Linx [En ligne],

10 | 1998, mis en ligne le 03 juillet 2012, consulté le 20 avril 2019. URL : http://

journals.openedition.org/linx/949; DOI : 10.4000/linx.949

Ce document a été généré automatiquement le 20 avril 2019.

Département de Sciences du langage, Université Paris Ouest 


\title{
Dicible et indicible : positions assumées par le sujet du discours ${ }^{1}$
}

\author{
Utterable and unutterable: roles played by the subject of discourse.
}

\section{Beth Brait}

1 Dans le chapitre "Le vocabulaire de la place publique dans l'oeuvre de Rabelais » (Bakhtine, 1970), l'auteur se réfère aux nombreuses expressions forgées par des critiques à différentes époques pour désigner certains aspects de l'oeuvre du créateur de Gargantua et Pantagruel, difficiles à assimiler. De façon plus spécifique, ces références désignent le vocabulaire, lequel « embarrasse tous les lecteurs ", parce qu'il caractérise le champ sémantique ayant pour référent la zone des organes génitaux, ce qu'on appelle «bas » corporel. C'est le cas, par exemple du moraliste La Bruyère, qui au XVII ème siècle, qualifiait ces aspects de «charme de la canaille » et de «sale corruption»; de Voltaire, qui au XVIII ${ }^{\text {ème }}$ les appelait «ramas d'impertinences et grossières ordures»; de George Sand qui, au XIX ${ }^{\text {ème }}$, voulait purement et simplement expurger l'oeuvre.

2 En attirant l'attention sur la disqualification de l'oeuvre de Rabelais par les critiques, Bakhtine vise justement à renverser cette situation en décaractérisant cette dimension de mauvais goût, de vulgarité qui lui est attribuée et en proposant une lecture contextuelle, c'est-à-dire une lecture qui tienne compte du sens des gestes et des expressions verbales correspondantes à l'époque de sa création. En analysant les spécificités du texte, de l'intertextualité et de l'interdiscursivité, l'auteur présente des arguments qui servent à montrer où réside la difficulté de compréhension de ces termes, considérés, depuis l'époque de La Bruyère, comme pornographie, cynisme scatologie, termes imprononçables.

3 Selon l'auteur, au Moyen Age et sous la Renaissance, ces termes étaient liés à la place publique de la ville, au domaine du marché, du carnaval et constituaient des formes de nature ambivalente, élaborées au long des siècles et qui articulaient la zone des organes génitaux, le bas, avec la fécondité, la naissance, le renouvellement et le bien-être. Bakhtine l'explique ainsi :

«Les éléments de la place publique (...) sont imprégnés dans toute leur diversité de

l'unité de la culture populaire du Moyen Age ; dans le livre de Rabelais, cette unité 
est organiquement alliée aux principes nouveaux de la Renaissance. Sous ce rapport, les prologues (...) démystifient les bases mêmes de la conception médiévale du monde, qu'ils refoulent dans le passé en même temps qu'ils regorgent d'allusions et échos de l'actualité politique et idéologique » (Bakhtine, 1970 ; 197).

4 Si nous considérons qu'au-delà des spécificités de l'oeuvre de Rabelais, les positions critiques négatives sont elles aussi liées à ce que chaque époque considère comme pertinent du point de vue d'une norme littéraire, d'une norme linguistique cultivée, de ce qu'on appelle le goût, nous pouvons compléter, si je puis dire, la perspective de Bakhtine, en orientant la discussion dans la direction de la classification du vocabulaire, de certains gestes et attitudes et mêmes de certains thèmes et sujets déterminés sur la liste des éléments qu'une époque donnée considère comme de bon ou de mauvais goût. C'est de ce point de vue que nous pouvons constater l'existence, jusqu'à nos jours, de limites très précises en ce qui concerne les expressions faisant une référence directe ou à peine une allusion à la zone et aux fonctions des organes génitaux.

$5 \quad \mathrm{Au}$ XVII ${ }^{\text {ème }}$ siècle, au moment où La Bruyère écrit son oeuvre célèbre, Les Caractères, il y avait, parmi les éléments constituant les normes littéraires de l'époque, la règle des convenances ou de la bienséance, comme on disait, qui a été définie pour les pièces de théâtre et plus particulièrement la tragédie classique de la manière suivante : « C'est une exigence morale qui demande que la pièce de théâtre ne choque pas le goût, les idées morales ou, si l'on veut, les préjugés du public (Jacques Scherer, apud Faria, 1995 : 13). Cela signifie qu' «Il y avait des choses qui ne pouvaient être dites ou montrées, au nom de la raison, du bon goût et de la morale chrétienne. Ainsi furent bannis de la scène les mots grossiers ou obscènes, qui apparaissaient parfois dans les tragi-comédies, de même que les scènes où les personnages mangent, boivent ou dorment » (Faria, 1995 : 13).

6 Mais si «la dramaturgie classique exprime une vision du monde en conformité avec le goût et les valeurs politiques et morales de la monarchie » (Faria : 17), on ne laisse pas de voir à la même époque, aussi bien du point de vue de la production que de celui de la critique, la présence d'autres formes littéraires situées en dehors des normes et bien souvent pratiquées par les mêmes écrivains consacrés.

Du point de vue de la création, il suffit de rappeler, par exemple, qu'à côté des fables didactiques et moralisantes, La Fontaine, écrivain typique du XVII ${ }^{\text {ème }}$ siècle, a écrit aussi des contes considérés comme tellement licencieux qu'ils furent interdits en 1674. Dans ses contes et nouvelles, parus entre 1665 et 1674, La Fontaine rétablit la vieille tradition des contes cocasses, qui réitèrent les thèmes des filles dépucelées, des maris trompés, des femmes infidèles et rusées, des moines débauchés qui se trouvaient déjà dans Boccace, dans Marguerite de Navarre, dans l'Arioste. Tout ceci traité évidemment avec un grand raffinement esthétique, dans un vocabulaire bien différent de celui des fables, mais où les moralistes n'ont vu que des écrits libertins, uniquement des attentats à la morale et au bon goût.

8 Au Brésil, nous pouvons évoquer plusieurs cas semblables, à savoir, d'auteurs bienséants, soumis au bon goût, aux normes esthétiques d'une époque et qui, parallèlement à ces créations on enregistré avec le vocabulaire de la place publique, si nous continuons à utiliser la métaphore de Bakhtine, les thèmes liés à l'imaginaire provocant, drôle et irrévérencieux peuplé par les organes génitaux et leurs fonctions.

9 C'est le cas de l'écrivain romantique Bernardo Guimarães, popularisé par son oeuvre abolitionniste L'Esclave Isaura, roman publié en 1875 et qui s'est transformé à notre époque, plus précisément dans les années 80 , en feuilleton télévisé à succès. Originaire 
d'Ouro Preto, dans l'Etat de Minas Gerais, et ayant fait ses études de Droit à la Faculté du "Largo São Francisco "², grand compagnon d'Álvares de Azevedo, Bernardo Guimarães s'est fait connaitre par sa prose romantique de caractère nationaliste, nourrie de thèmes historiques, indianistes et régionalistes. Même la sensualité qui affleure dans son oeuvre Le Séminariste, peut-être la meilleure de sa production, ne laisse pas deviner l'auteur de Elixir do Pajé, A Origem do Mênstruo et l'Orgia dos Duendes ${ }^{3}$.

$\mathrm{Si}$, en ce qui concerne l'ensemble de son oeuvre, la critique se plaint de son langage ingénu, trop soumis aux conventions du citadin par rapport à la campagne, dilué dans des descriptions romantiques conventionnelles, c'est certainement cette même critique qui, rougissant au langage du chaman, l'écarte des histoires de la littérature, des recueils didactiques et scolaires et des anthologies poétiques, comme ces paroles d'Alphonsus de Guimarães Filho en font foi, dans l'introduction aux Poésies Complètes: "Nous sommes allés le chercher là pour cette édition, qui présente tout, sauf évidemment les poèmes érotiques de Bernardo Guimarães (Guimarães, 1959 ; XVI) ».

Ici non plus, dans cet espace académique, je ne pourrais lire un seul vers de L'Elixir $d u$ Chaman sans que cette attitude ne semble d'un profond mauvais goût, et ne cause pour moi et pour tous ceux qui m'écoutent, un grand embarras. Dans cette situation spécifique, ce sont des mots imprononçables. Cela ne veut pas dire que je ne pourrais pas les prononcer, devant les mêmes personnes qui se trouvent ici, dans une autre situation, dans un autre espace ou que je ne pourrais pas analyser ces vers par écrit, dans leur dimension de discours d'affrontement aux discours officiels.

Dans notre espace académique, le commentaire que je pourrais faire, en demeurant dans les limites du dicible, du bon goût, de façon à communiquer le contenu et les caractéristiques du texte, devrait être soumis à une traduction du vocabulaire ce qui donnerait à peu près le résultat suivant: L'Elixir du Chaman est une poème narratif dont les principaux personnages sont une personnalité indigène qui a des problèmes d'impuissance, son organe sexuel et une potion magique qui lui rend ses pouvoirs masculins. Si l'on considère que l'auteur est à la fois un romantique nationaliste et un bohème, fondateur de la société Epicureia, espace où il présentait ses «bestialogues $»^{4}$, ses "curieuses extravagances rimées d'un humour quelque peu surréaliste », on peut remarquer, d'après l'irrévérence du thème, des personnages et du vocabulaire, non seulement la rupture des tabous linguistiques, mais également celle des tabous mythiques de la nationalité. Dans la mesure où le thème de l'indien si cher au romantisme brésilien et à la tradition du bon sauvage, est récupéré d'une façon irrévérencieuse, en échangeant l'appareil figuratif $\mathrm{du}$ brave, fort, viril et pur aborigène contre l'esquisse du libertin impuissant, la parodie interdiscursive se trouve configurée, selon un point de vue critique de la norme littéraire en vigueur. C'est ce que nous appellerions aujourd'hui une position " politiquement incorrecte ", de très mauvais goût, mais extrêmement critique et drôle.

Montaigne lui-même dans ses Essais, au chapitre «Sur des vers de Virgile », a abordé cette question des mots relatifs aux organes génitaux:

Qu'a fait l'action génitale aux hommes, si naturelle, si nécessaire et si juste, pour n'en oser parler sans vergogne et pour l'exclure des propos sérieux et réglés ? Nous prononçons hardiment : tuer, dérober, trahir ; et cela, nous n'oserions qu'entre les dents ? Est-ce à dire que moins nous en exhalons en paroles, d'autant nous avons loi d'en grossir la pensée?

Car il est bon que les mots qui sont le moins en usage, moins écrits et mieux tus, sont les mieux sus et plus généralement connus (...) Ils s'impriment en chacun de nous sans être exprimés et sans voix et sans figure. Il est bon aussi que c'est une 
action que nous avons mise en la franchise du silence, d'où c'est crime de l'arracher

(...) (Montaigne, $1967: 343)$ dans ses aspects opposés « bon goût » et " mauvais goût », comme une production de sens et d'effets de sens ayant lieu à partir de catégories qui embrassent en même temps des modèles éthiques, esthétiques, sociaux, culturels et, également, subjectifs lesquels, même s'ils varient dans le temps, placent le vocabulaire et les thèmes rattachés à la sexualité dans un champ d'interdiction parfaitement délimité. Le silence et la passivité dans lesquels ils restent maintenus peuvent être rompus dans des circonstances spéciales qui révèlent de façon discursive l'existence de cette dimension. Lorsque cela se produit, et cela se produit avec une fréquence bien plus grande que le bon goût ne le prévoit, quoi qu'on entende par là à chaque époque, ce qu'on a, par la transgression, c'est l'exposition de différents modes de représentations de l'interdiscours, l'hétérogénéité constitutive de toute société.

Rabelais, par exemple, qui connaissait très bien la vie sur le terrain de la foire, récupère le vocabulaire de la place publique au sens exposé par Bakhtine, c'est-à-dire sous les formes que le langage populaire trouvait pour s'infiltrer dans les genres liés aux festivités, qui gravitaient autour de la place, où ils jouissaient d'un droit que l'auteur dénomme « droit d'exterritorialité dans le monde de l'ordre et de l'idéologie officiels» (Bakhtine, 1970 : 156). Il est bien vrai, comme l'affirmait également le penseur russe, que ces discours qui retentissaient en place publique, formaient presque une espèce de langage familier, une langue spéciale, inutilisable ailleurs, nettement différenciée de celle de l'Eglise, de la cour, des tribunaux, des institutions publiques, de la littérature officielle, de la langue parlée des classes dominantes (...) bien que le vocabulaire de la place publique y fit de temps à autre irruption sous certaines conditions. Les jours de fête, surtout pendant le carnaval, le vocabulaire de la place publique s'insinuait partout, dans une mesure plus ou moins grande, y compris dans l'église (...) (Bakhtine, 1970 : 157).

16 Il en va de même pour le brésilien Bernardo Guimarães. Sans la grandeur de Rabelais, il se fit connaître et apprécier pour sa prose et sa poésie de goût fortement romantique. Mais par les voies transgressives de la poésie érotique, considérée non pas d'un goût douteux, mais d'une impertinence de vocabulaire capable d'empêcher sa lecture publique jusqu'à aujourd'hui, il a récupéré pour la production littéraire brésilienne un discours extraofficiel, un bêtisier apparemment gratuit et grossier, mais dont la fonction est, à partir de l'humour, d'enregistrer l'envers du discours romantique officiel et qui fait partie autant que ce dernier de l'ensemble des discours fondateurs de la nationalité. La figure de l'indien, les vers rythmés et bien mesurés, les allusions à la nature, à l'intertextualité avec des vers connus de la poésie romantique brésilienne, auraient pu constituer un ensemble de vers officiels de plus si ce n'était le thème scabreux et destructeur du mythe, réalisé dans un vocabulaire directement lié aux organes génitaux.

Le grotesque, le vulgaire, ce qui est de mauvais goût au sens éthique ou même esthétique du mot est en général un discours qui s'oppose aux discours officiels, à différentes normes reconnues et pratiquées à un moment donné. C'est ce qui rompt la superficie de la norme et met à nu ses domaines souterrains latents. L'exemple de la littérature sert ici à montrer un discours capable de créer les conditions pour ce lever de masque, quoique cette rupture se produise toujours dans des conditions spéciales et s'insère immédiatement dans une tradition de l'irrévérence, de la transgression. 

dont la nature ne peut être reconnue que dans ce que Bakhtine a appelé " exterritorialité », un territoire socialement établi pour ce type de langage, dans d'autres discours que le discours littéraire, toujours dans ce lacis formé entre les différents paramètres de ce qui est éthique et de ce qui est esthétique. La conséquence en est l'occupation d'un espace qui n'est pas le sien à l'origine, même si cette occupation est autant que possible repoussée par les normes tenues pour de bon goût et de bon ton. Une lutte du même type a lieu, par exemple, entre la norme dite cultivée et les autres variantes linguistiques coexistantes à un moment donné, ou même dans le discours raciste, qui dans une société qui se dit non raciste, apparaît sous la forme de blagues. Mamonas Assassinas ${ }^{5}$, et si nous tenons compte du fait qu'on la joue tous le jours et à n'importe quelle heure à la radio, sur des émetteurs qui ne sont pas particulièrement populaires, mais sont aussi écoutés par la classe moyenne et moyenne supérieure, nous avons un bel exemple d'un discours musical parodique que l'on peut considérer d'un extrême mauvais goût et politiquement incorrect si on le considère d'un point de vue éthique, mais qui en réalité transporte dans un nouvel espace, celui des médias institutionnelles, un discours de place publique, de marché, de trottoir. Ce discours est reconnu même par ceux qui ne fréquentent pas ces espaces, bien qu'ils cohabitent avec ceux-ci de différentes manières, comme faisant partie de l'univers discursif de la société brésilienne.

20 A la différence de Bernardo Guimarães, Olavo Bilac, Carlos Drummond de Andrade, Adélia Prado et d'autres écrivains insérés dans le bon goût de l'époque et disposant peut-être pour cette raison de droit et d'outils discursifs pour le subvertir, les membres du groupe Mamonas Assassinas, que cela nous plaise ou non, font irruption, en provenance directe de l'extraterritorial pour être consommés institutionnellement. Si ce phénomène est une création des médias, cela ne change rien au fait sémiotiquement constatable que les différents discours qui constituent l'interdiscours social, et qui caractérisent les différentes sources de l'hétérogénéité du langage, se déplacent de façon à disputer les lieux institutionnalisés. Rabelais n'a pas écrit pour demeurer dans l'oubli. Bernardo Guimarães non plus.

\section{BIBLIOGRAPHIE}

BAKHTINE, M. (1970) L'oeuvre de François Rabelais et la culture populaire au Moyen Age et sous la Renaissance, Trad. A. Robel, Paris, Gallimard.

FARIA, J.-R. « A dramaturgia do classismo ». In O Classicismo, São Paulo, Perspectiva (en préparation).

GUIMARÃES, B. (1959) Poesias completas de Bernardo Guimarães, Rio de Janeiro, MEC/ INL.

GUIMARÃES, B. (1992) Poesia erótica e satírica, Rio, Imago.

LA FONTAINE. (s/d) Contes et nouvelles, Paris, Librairie Gründ. 
MONTAIGNE. (1967) Oeuvres complètes, Paris, Seuil.

\section{NOTES}

1. Ce texte tient à conserver les conditions de sa présentation, à savoir, les circonstances académiques d'un séminaire, directement liées à la question du dicible et de l'indicible. Il fait partie, avec quelques modifications, de l'oeuvre 0 gosto da gente, o gosto das coisas - Le goût des gens, le goût des choses (São Paulo, EDUC, 1997).

2. Nom donné à São Paulo à la Faculté de Droit, du nom de la place où elle est située. Fondée en 1828.

3. «L'Elixir du Chaman », «L'Origine des Menstrues », "L'Orgie des Farfadets ». Pajé : mot de la langue tupi, qui désigne le chef spirituel, prophète et médecin guérisseur d'une tribu indigène.

4. Recueils de textes incongrus, de coq à l'âne.

5. « Ricins Assassins » c'est le nom d'un groupe musical qui composait des chansons dans un style très irrévérencieux et dans un vocabulaire très grossier. Ils remportèrent rapidement un grand succès, surtout auprès des jeunes et même des enfants, en 1996, avant de mourir dans un accident d'avion. Voici des extraits de la chanson "vira-vira ", difficiles à traduire à cause des doubles sens, approximativement:

« Partouge », « Toute retroussée elle ne pouvait même pas s'asseoir »

"Ils m'ont mis la main au cul / et je ne me suis même tapé personne »

«Manuel, t'as de la merde dans la tête / Laisse tomber la putasserie et occupe toi d'la boulangerie (fesses)».

\section{RÉSUMÉS}

Cet article se propose de travailler la question du dicible et de l'indicible comme des positions assumées par le sujet du discours dans différentes situations d'énonciation. Pour développer quelques points de cette question, on a choisi les conditions de production et de réception des oeuvres d'un écrivain brésilien, Bernardo Guimaraens (1825-1884), connu pour sa prose romantique de caractère nationaliste, nourrie de thèmes historiques, indianistes et régionalistes. $\mathrm{Si}$, en ce qui concerne l'ensemble de son oeuvre, la critique se plaint de son langage naif, trop soumis aux conventions du citadin par rapport à la campagne, et dilué dans des descriptions romantiques conventionnelles, c'est certainement cette même critique qui, rougissant au langage du chaman, l'écarte des histoires de la littérature, des recueils didactiques et scolaires, des anthologies poétiques. C'est à partir des concepts de sujet, langage et énonciation du théoricien russe Mikhail Bakhtine que l'on a pu faire une réflexion sur les positions discursives, toujours très diverses et polémiques, assumées par le sujet.

The purpose of this paper is to address the question of what can be said utterable or unutterable ( indicible) based on the roles played by the subject of discourse in utterance situations (situations d'énonciation). In order to develop some points concerning this issue, the conditions of production and reception of the work of a Brazilian writer (Bernardo Guimaraens, 18825-18884), have been chosen.This author is kwnown for his romantic prose with a nationalist quality, characterized by historical, indianist and regionalist themes. Despite the fact that critics resset, about his work as 
a whole, its naive language, cluttered with the conventions of the city as opposed to the countryside, thes same critics, reacting to the language of his novel Elixir do Paje, include it neither in the histories of literature nor in the didactic and school anthologies. Using concepts of subject, language, and utterance (énonciation) proposed by the Russian theorist Mikhail Bakhtin, the different and polemic discursive roles played by the subject can be discussed.

\section{AUTEUR}

\section{BETH BRAIT}

Université de São-Paulo (USP) 